\title{
ON RAMIFICATION LOCUS OF A POLYNOMIAL MAPPING
}

\author{
ZBIGNIEW JELONEK \\ Institute of Mathematics, Polish Academy of Sciences \\ Św. Tomasza 30, 31-027 Kraków, Poland \\ E-mail: najelone@cyf-kr.edu.pl
}

\begin{abstract}
Let $X$ be a smooth algebraic hypersurface in $\mathbb{C}^{n}$. There is a proper polynomial mapping $F: \mathbb{C}^{n} \rightarrow \mathbb{C}^{n}$, such that the set of ramification values of $F$ contains the hypersurface $X$.
\end{abstract}

Let $F=\left(F_{1}, \ldots, F_{n}\right): \mathbb{C}^{n} \rightarrow \mathbb{C}^{n}$ be a polynomial proper mapping. We say that a point $y \in \mathbb{C}^{n}$ is a ramification value of $F$ if $\# F^{-1}(y)<\mu(F)$, where $\mu(F)=\left(\mathbb{C}\left(x_{1}, \ldots, x_{n}\right)\right.$ : $\left.\mathbb{C}\left(F_{1}, \ldots, F_{n}\right)\right)$ denotes the geometric degree of $F$. The set of all ramification values of $F$ (which always is a hypersurface or the empty set) is called the ramification locus of $F$. This set will be denoted by $R L(F)$. Some properties of this set were studied in [1] and [2].

In particular in the paper [2] we showed that for a given hypersurface $X \subset \mathbb{C}^{n}$ there is a proper polynomial mapping $F: \mathbb{C}^{n} \rightarrow \mathbb{C}^{n}$ and a hypersurface $X^{\prime}$, which is birationally equivalent to $X$, such that $X^{\prime} \subset R L(F)$.

However sometimes it is interesting to know whether a hypersurface $X$ itself is a component of the set $R L(F)$ (for some proper mapping $F$ ). In particular Professor R. V. Gurjar asked whether the hyperbola $\Gamma=\left\{(x, y) \in \mathbb{C}^{2}: x y=1\right\}$ can be a component of the set $R L(F)$ (for a proper mapping $F: \mathbb{C}^{2} \rightarrow \mathbb{C}^{2}$ ). In this note we give the following general answer to this question.

TheOREM 1. Let $X$ be a smooth algebraic hypersurface in $\mathbb{C}^{n}$. There is a proper polynomial mapping $F: \mathbb{C}^{n} \rightarrow \mathbb{C}^{n}$, such that $X \subset R L(F)$.

Proof. Of course, we can assume that $n>1$. First we construct a mapping $G: \mathbb{C}^{n} \rightarrow$ $\mathbb{C}^{n}$ such that

a) $G\left(x_{1}, \ldots, x_{n}\right)=\left(x_{1}, \ldots, x_{n}\right)$ on $X$,

b) $\operatorname{Jac}(G)(x)=0$ for $x \in X$.

2000 Mathematics Subject Classification: Primary 14D06; Secondary 14Q20.

Research supported by KBN grant 2P03A 01722.

The paper is in final form and no version of it will be published elsewhere. 
Let $f=0$ be a reduced equation for $X$. Since $X$ is smooth, polynomials $\frac{\partial f}{\partial x_{1}}, \ldots, \frac{\partial f}{\partial x_{n}}$ have no common zeroes on $X$. Let $f_{i}=\frac{\partial f}{\partial x_{i}}$. We look for $G$ among mappings of the form $G=\left(x_{1}+\alpha_{1} f, \ldots, x_{n}+\alpha_{n} f\right)$. We choose $\alpha_{i}$ in such a way that condition b) be satisfied. It is easy to see that on $X$ the Jacobian of $G$ is equal to

$$
\left|\begin{array}{cccc}
1+\alpha_{1} f_{1} & \alpha_{1} f_{2} & \ldots & \alpha_{1} f_{n} \\
\alpha_{2} f_{1} & 1+\alpha_{2} f_{2} & \ldots & \alpha_{2} f_{n} \\
\vdots & \vdots & \ddots & \vdots \\
\alpha_{n} f_{1} & \alpha_{n} f_{2} & \ldots & 1+\alpha_{n} f_{n}
\end{array}\right|=1+\sum_{i=1}^{n} \alpha_{i} f_{i} .
$$

Since polynomials $f_{1}, \ldots, f_{n}$ have no common zeroes on $X$, by the Hilbert Nullstellensatz there exist polynomials $A_{i}$ such that

$$
1=\sum_{i=1}^{n} A_{i} f_{i} \quad \text { on } X .
$$

Take $\alpha_{i}=-A_{i}$. Of course the mapping $G=\left(x_{1}+\alpha_{1} f, \ldots, x_{n}+\alpha_{n} f\right)$ satisfies conditions a) and b). Let $G=\left(G_{1}, \ldots, G_{n}\right)$ and let $g$ denote the restriction of the mapping $G$ to $X$. The mapping $g$ is the identity (on $X$ ), this implies that polynomials $x_{i}-G_{i}$ vanish on $X$. Take

$$
\Phi: \mathbb{C}^{n} \ni x \rightarrow\left(G(x),\left(x_{1}-G_{1}\right)^{2}, \ldots,\left(x_{n}-G_{n}\right)^{2}\right) \in \mathbb{C}^{n} \times \mathbb{C}^{n} .
$$

By construction the mapping $\Phi$ is finite, $\Phi(X) \subset \mathbb{C}^{n} \times\{0\}$ and $\operatorname{dim} \Phi\left(\mathbb{C}^{n}\right)=n$. Moreover, we have $d_{x}(\Phi)=\left(d_{x} G, 0\right)$ for $x \in X$. Let $\pi: \Phi(X) \rightarrow \mathbb{C}^{n} \times\{0\}$ be a generic linear projection and let $F=\pi \circ \Phi$. Then the mapping $F$ is finite and $F(x)=G(x), d_{x} F=d_{x} G$, for $x \in X$. In particular the mapping $F$ ramifies on $X$ and $F(X)=X$. This means that $X \subset R L(F)$.

EXAmple 1. In the case of the hyperbola $\Gamma=\left\{(x, y) \in \mathbb{C}^{2}: x y=1\right\}$ our method gives:

$$
F(x, y)=\left(x+\left(x-x^{2} y\right), y+\left(x-x^{2} y\right)^{2}\right) .
$$

In fact it is not difficult to check directly that the mapping $F$ is proper and $\Gamma \subset R L(F)$.

Question. Let $X$ be an arbitrary hypersurface in $\mathbb{C}^{n}$. Is it true that there exists a proper polynomial mapping $F: \mathbb{C}^{n} \rightarrow \mathbb{C}^{n}$ such that $X \subset R L(F)$ ?

Acknowledgements. The author wishes to express his thanks to Professor R. V. Gurjar for suggesting the problem.

\section{References}

[1] R. V. Gurjar, On ramification of self-maps of $\mathbb{P}^{2}$, J. Algebra 259 (2003), 191-200.

[2] Z. Jelonek, On ramification values of a polynomial mapping, Manuscripta Math. 109 (2002), 147-149. 